\title{
Electrical somatosensory stimulation followed by motor training of the paretic upper limb in acute stroke: study protocol for a randomized controlled trial
}

Emma Ghaziani ${ }^{1,2^{*}}$ (1) Christian Couppé ${ }^{1,3,4}$, Cecilie Henkel ${ }^{2}$, Volkert Siersma ${ }^{5}$, Mette Søndergaard ${ }^{6}$, Hanne Christensen ${ }^{2,6}$ and S. Peter Magnusson 1,3,4

\begin{abstract}
Background: Upper limb paresis is one of the most frequent and persistent impairments following stroke. Only $12-$ $34 \%$ of stroke patients achieve full recovery of upper limb functioning, which seems to be required to habitually use the affected arm in daily tasks. Although the recovery of upper limb functioning is most pronounced during the first 4 weeks post stroke, there are few studies investigating the effect of rehabilitation during this critical time window. The purpose of this trial is to determine the effect of electrical somatosensory stimulation (ESS) initiated in the acute stroke phase on the recovery of upper limb functioning in a nonselected sample of stroke patients.

Methods/design: A sample of 102 patients with upper limb paresis of varying degrees of severity is assigned to either the intervention or the control group using stratified random sampling. The intervention group receives ESS plus usual rehabilitation and the control group receives sham ESS plus usual rehabilitation. The intervention is applied as $1 \mathrm{~h}$ of ESS/sham ESS daily, followed by motor training of the affected upper limb. The ESS/sham ESS treatment is initiated within 7 days from stroke onset and it is delivered during hospitalization, but no longer than 4 weeks post stroke. The primary outcome is hand dexterity assessed by the Box and Block Test; secondary outcomes are the Fugl-Meyer Assessment, hand grip strength, pinch strength, perceptual threshold of touch, degree of pain, and modified Rankin Scale score. Outcome measurements are conducted at baseline, post intervention and at 6-month follow-up.

Discussion: Because of the wide inclusion criteria, we believe that the results can be generalized to the larger population of patients with a first-ever stroke who present with an upper limb paresis of varying severity. On the other hand, the sample size $(n=102)$ may preclude subgroup analyses in such a heterogeneous sample. The sham ESS treatment totals a mere $2 \%$ of the active ESS treatment delivered to the intervention group per ESS session, and we consider that this dose is too small to induce a treatment effect.
\end{abstract}

Trial registration: ClinicalTrials.gov, NCT02250365. Registered on 18 September 2014.

Keywords: Acute stroke, Upper extremity, Recovery of function, Electrical stimulation, Rehabilitation, Longitudinal studies

\footnotetext{
* Correspondence: emma.ghaziani@regionh.dk

${ }^{1}$ Department of Physical and Occupational Therapy, Bispebjerg Hospital,

Bispebjerg Bakke 23, bldg. 10, 2400, Copenhagen, Denmark

${ }^{2}$ Faculty of Health and Medical Sciences, University of Copenhagen,

Copenhagen, Denmark

Full list of author information is available at the end of the article
} 


\section{Background}

Stroke is ranked as the third largest cause of disease burden globally [1], causing substantial physical, psychological and financial demands on patients, families, and societies at large [2-4]. Upper limb paresis is one of the most frequent impairments following stroke and affects 48-77\% of patients in the acute stroke phase [5-7]. Moreover, upper limb paresis has been identified as a major obstacle to regaining independence in activities of daily living (ADLs) [8]. In fact, only $12-34 \%$ of the patients achieve full functional recovery of the affected upper limb at 6 months post stroke $[9,10]$. This represents a considerable challenge since near complete functional recovery is required to routinely involve the affected upper limb in performing ADLs [11].

Recovery of upper limb functioning is typically pronounced during the first month and subsequently levels off by 6 months post stroke [12-14]. Regaining hand dexterity (i.e., motor skills such as reaching, grasping, gripping, moving and releasing objects) is often achieved already within the first 4 weeks, implying that there may be a critical time window for recovery of upper limb functioning $[9,10]$ during which rehabilitation efforts may maximize functional recovery. However, there are few studies investigating the effect of motor rehabilitation methods in the initial weeks after stroke.

Electrical stimulation (ES) is one of the methods that have been used to facilitate recovery of upper limb functioning following stroke. ES can induce a muscle contraction, or it can be a somatosensory stimulation below the motor threshold [15]. The majority of studies using ES have been conducted in chronic stroke and, therefore, it remains unknown to what extent ES applied in the acute phase after stroke could affect the recovery of upper limb functioning. Also, these investigations have largely focused on ES that induces muscle contraction. In healthy persons, the application of low-intensity ES with no or small motor responses to peripheral hand nerves [16-20], forearm muscles [21] or the whole hand $[22,23]$ elicits an increase in the cortical excitability of the representations that control the stimulated body parts, which seems to outlast the stimulation period itself $[18,21,23]$. It has been hypothesized that increasing the amount of somatosensory input may enhance the motor recovery of patients following stroke [24]. Recent data on acute, subacute and mostly chronic stroke patients suggest that a single 2-h session of ESS to the peripheral hand nerves leads to transient improvement of pinch force, movement kinematics and upper limb motor skills required for ADL performance [25-31]. However, ESS was only used in conjunction with motor training in one of these studies [29]. Interestingly, there is some evidence that multiple sessions of ESS to the peripheral hand nerves, in conjunction with motor training, might improve motor skills of the paretic upper limb in subacute [32, 33] and chronic stroke patients [34], and, moreover, that these positive results seems to be long lasting [34]. However, the effect of ESS in conjunction with motor training has never been investigated in acute stroke patients. It is noteworthy that ESS is benign in nature, causes patients minimal discomfort and adverse effects (itch and blushing), is relatively inexpensive and can easily be incorporated into clinical practice [35]. Therefore, it would be valuable to establish the effect of multiple sessions of ESS in conjunction with motor training in the restoration of upper limb functioning in the acute stroke phase.

The purpose of the present trial is to investigate the effect of multiple sessions of ESS treatment accompanied by motor training on the recovery of the affected upper limb following stroke. The ESS treatment is initiated in the acute stroke phase and each ESS session is immediately followed by motor training of the paretic upper limb. Specifically, we wish to address the following:

(1)Does ESS treatment: (a) reduce motor and sensory impairments, (b) improve hand dexterity and (c) reduce disability at the end of the intervention period (short-term effect)?

(2)Are the changes that can be observed at the end of the intervention period still present or improved at 6 months post stroke (long-term effect)?

Our hypothesis is that ESS treatment initiated in the acute stroke phase will improve paretic upper limb functioning as measured by the Box and Block Test (BBT) (primary outcome measure) at 6 months post stroke.

\section{Methods/design \\ Trial design}

This study is conducted as a single-blinded randomized controlled trial with two arms and blinded endpoint adjudication. The intervention consists of ESS/sham ESS treatment immediately followed by training of the affected upper limb in addition to usual rehabilitation. The ESS/sham ESS treatment is initiated within the first 7 days post stroke. The first 4 weeks post stroke seem to be crucial for gaining maximal recovery of upper limb functioning $[9,10]$. Since it is not possible for us to continue the intervention after hospital discharge for financial and logistical reasons, we decided to investigate the effect of our intervention during hospital stay (mean hospital stay when designing this study: 21 days), but no longer than 4 weeks post stroke. Outcome measures are assessed at three time points: (1) within the first 7 days post stroke prior to intervention onset 
(baseline), (2) at hospital discharge or 4 weeks post stroke (post-intervention) and (3) at 6 months post stroke (follow-up), which is the time point where the recovery of upper limb functioning is expected to level off [12-14]. Figure 1 shows the SPIRIT flow diagram of the trial.

\section{Trial setting and participants}

Trial participants are recruited among patients admitted to the stroke unit (consisting of an acute unit and a rehabilitation unit) at Bispebjerg Hospital, which is one of the university hospitals in the Capital Region of Denmark. The hospital currently serves a welldefined urban catchment area with a population of approximately 400,000 citizens from the Municipality of Frederiksberg and the larger part of Municipality of Copenhagen for stroke rehabilitation [36]. Except for holiday periods and periods of recruitment of new trial personnel, all patients consecutively admitted to

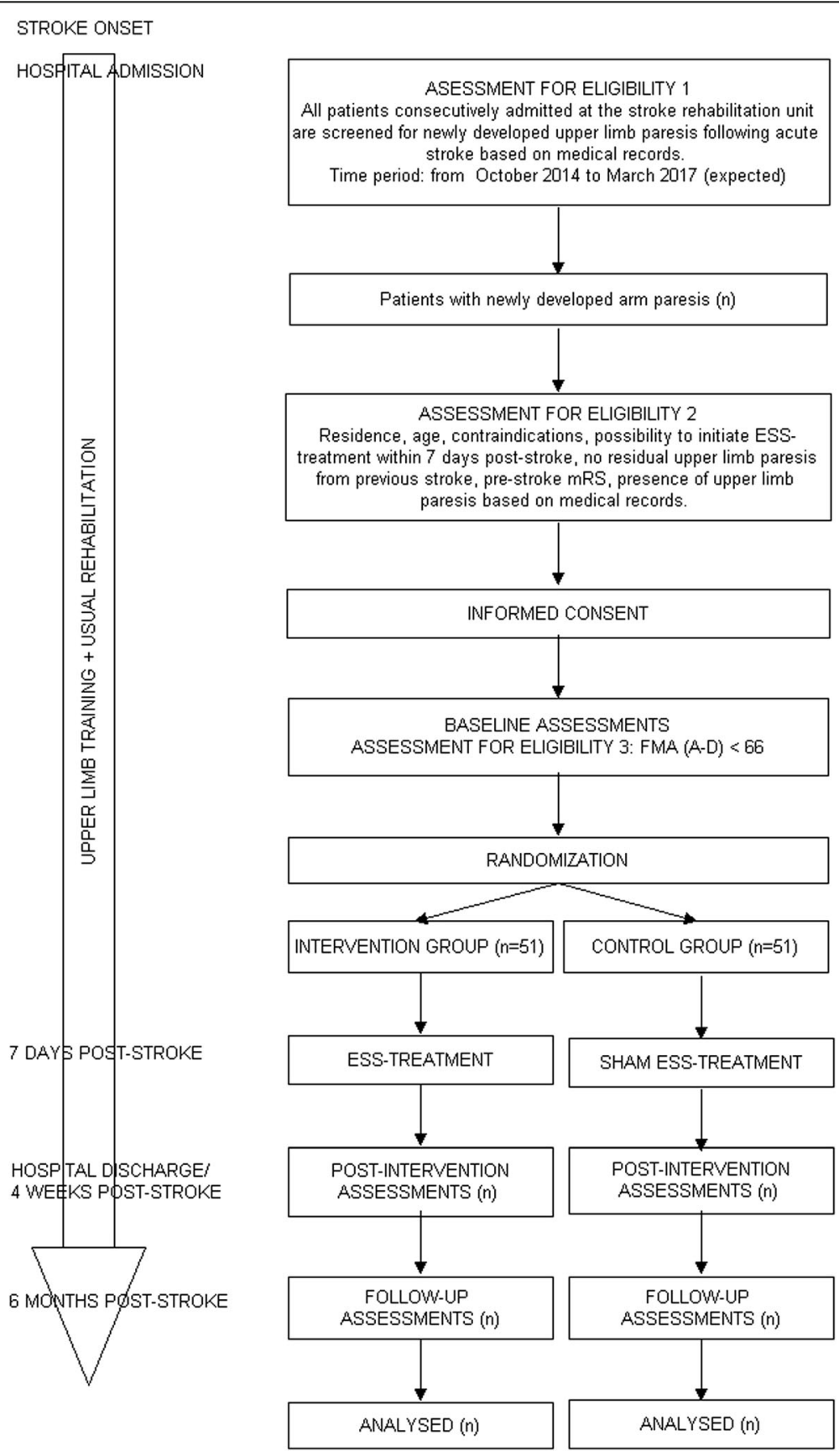

Fig. 1 SPIRIT flow diagram of the trial 
the stroke unit are assessed for eligibility by EG according to following inclusion criteria:

1. Admission at the rehabilitation stroke unit

2. Diagnosis of acute stroke confirmed by magnetic resonance imaging (MRI) or computed tomography (CT) scan

3. Upper limb paresis as indicated by a subscore $<66$ on the upper limb section of the Fugl-Meyer Assessment (FMA) (A-D) (see "Outcome measures" section)

4. Residence in the hospital's catchment area

5. Aged 18 years or older

Patients are not included if any of the following exclusion criteria are present:

1. Contraindications to ESS (e.g., pacemaker in situ, skin impairment) [37]

2. Inability to initiate the ESS treatment within 7 days post stroke

3. Presence of cognitive dysfunctions or poor communication skills in Danish that limits the ability to provide informed consent

4. Severe prestroke disability as indicated by a modified Rankin Scale (mRS) score $=5$ (see "Outcome measures" section)

5. Incomplete recovery of the affected upper limb after a previous stroke

6. Participation in other biomedical intervention trials within the last 3 months

The recruitment process takes place in several steps which are presented in the flow diagram in Fig. 1.
We are collecting data on the following variables to characterize the sample and to assess whether the randomization is successful: (a) age, (b) gender, (c) profession, (d) living condition (i.e., married or living with another person, alone), (e) risk factors for stroke: smoking, alcohol consumption, prestroke nutritional habits, prestroke physical activity level, weight, height, Body Mass Index, waist circumference and other risk factors for stroke (e.g., previous stroke, hypertension, hyperlipidemia, heart disease), (f) type of stroke (i.e., hemorrhage or infarction), (g) subtype of ischemic stroke [38], (h) severity of stroke at baseline [39], (i) stroke acute treatment, (j) affected upper limb, (k) dominant hand, (l) medications, $(\mathrm{m})$ complications during hospitalization, (n) amount and content of upper limb training following ESS and (o) amount and content of usual rehabilitation. For further information, see Table 1.

\section{Intervention}

The ESS treatment is administered at the rehabilitation stroke unit. Trained health care personnel who are not involved in usual rehabilitation or outcome assessments initiate the ESS treatment within 7 days post stroke according to a standardized protocol. Both groups receive $1 \mathrm{~h}$ of daily ESS from Monday to Sunday throughout their hospital stay, but for no longer than 4 weeks post stroke. Cortical excitability in healthy persons increases after $2 \mathrm{~h}$ of suprasensory ESS of peripheral hand nerves at the wrist $[18,20]$, but it is sufficient with $30 \mathrm{~min}$ of suprasensory ESS of the whole hand to induce increased cortical excitability that lasts for $1 \mathrm{~h}$ after stimulation has ended [22, 23]. When electrical stimulation is applied for a longer time period, it seems that cortical

Table 1 Time schedule of enrollment, intervention, assessments and responsible trial personnel

\begin{tabular}{|c|c|c|c|c|c|c|c|}
\hline \multirow[t]{2}{*}{ Trial procedure } & \multicolumn{5}{|c|}{ Time points during the course of the trial } & \multirow[t]{2}{*}{ Responsible personnel } & \multirow{2}{*}{$\begin{array}{l}\text { Blinded to } \\
\text { group allocation? }\end{array}$} \\
\hline & $\begin{array}{l}\text { Stroke onset - } 7 \text { days } \\
\text { post stroke }\end{array}$ & ESS treatment & Post- intervention & $\begin{array}{l}\text { Hospital } \\
\text { discharge }\end{array}$ & $\begin{array}{l}\text { 6-month } \\
\text { follow-up }\end{array}$ & & \\
\hline Assess eligibility & $x$ & & & & & EG & Not applicable \\
\hline $\begin{array}{l}\text { Present trial information for } \\
\text { potential participants }\end{array}$ & $x$ & & & & & EG/ESS personnel & Not applicable \\
\hline Collect informed consent & $x$ & & & & & EG & Not applicable \\
\hline Group allocation & $x$ & & & & & $\begin{array}{l}\text { Administrative } \\
\text { personnel/SPM }\end{array}$ & $\mathrm{No} / \mathrm{no}$ \\
\hline Assess outcome & $x$ & & $x$ & & $x$ & EG & Yes \\
\hline Collect sociodemographic data & $x$ & & & $x$ & $x$ & EG & Yes \\
\hline Collect medical data & $x$ & & & $x$ & $x$ & MS/EG/ESS personnel & Yes/yes/no \\
\hline $\begin{array}{l}\text { Collect data on prestroke } \\
\text { nutritional habits and physical } \\
\text { activity }\end{array}$ & $x$ & & & & & EG/ESS personnel & Yes/no \\
\hline $\begin{array}{l}\text { Collect data on upper limb } \\
\text { training and usual rehabilitation }\end{array}$ & & $x$ & & & & $\begin{array}{l}\text { OTs or PTs at the stroke } \\
\text { unit and in the community }\end{array}$ & Yes \\
\hline $\begin{array}{l}\text { Collect data on ESS treatment, } \\
\text { including adverse events }\end{array}$ & & $x$ & & & & ESS personnel & No \\
\hline
\end{tabular}

EG Emma Ghaziani, ESS electrical somatosensory stimulation, MS Mette Søndergaard, OT occupational therapist, PT physiotherapist, SPM S. Peter Magnusson 
excitability reaches a plateau after $45 \mathrm{~min}$ [19] and, therefore, we decided to provide the ESS treatment for 1 h. A Cefar Compex Theta 500 electrical device specially programmed to deliver ESS/sham ESS is used (DJO Global Switzerland Sàrl, Ch. du Dévent, Z.I. Larges Pièces A, 1024 Ecublens, Switzerland). Two sets of large electrodes (Performance electrodes, $10 \times 5 \mathrm{~cm}$, One Snap, DJO Nordic, AB., Murmansgatan 126, 212 25, Malmö, Sweden) are placed on the affected arm as follows (see Fig. 2):

1. One set of electrodes on the upper arm - one electrode on the front of the shoulder and the other on the back of the shoulder, both of them covering the lower part of the deltoid muscle

2. One set of electrodes on the forearm - one electrode just distal to the elbow crease, centered on the anterior aspect of the forearm; the other electrode just proximal to the palm of the hand on the anterior and radial aspect of the forearm

The stimulation level is determined individually for each patient in the beginning of each ESS session. Halfway into each ESS session, the stimulation level is adjusted, if necessary, in the intervention group, whereas the participants in the control group receive a short visit from the ESS personnel in order to ensure that the same amount of attention is given to both groups.

The intervention group receives suprasensory ESS delivered in a continuous mode (pulse width $=250 \mu \mathrm{s}$, frequency $=10 \mathrm{~Hz}$ ). Suprasensory ESS is defined as the highest current amplitude that elicits paresthesia without



Fig. 2 Placement of the electrodes any discomfort, pain or visible muscle twitches. Sham ESS is suprasensory ESS delivered in an intermittent mode (active stimulation intervals of $3 \mathrm{~s}$ are delivered in loops of $2.5 \mathrm{~min}$, pulse width $=250 \mu \mathrm{s}$, frequency $=10 \mathrm{~Hz}$ ). In this way the control group receives a total dose corresponding to $2 \%$ of the amount of active ESS delivered to the intervention group per ESS session, and we consider this dose to be too small to induce treatment effect. Apart from ESS/sham ESS during the hospital stay, no other electrical therapy is permitted to the affected upper limb during the 6-month trial period. Delivering other types of interventions (e.g., cognitive or motor training) is not allowed during the ESS sessions. For further details, see the ESS treatment protocol in Additional file 1.

The ESS/sham ESS treatment is followed by motor training of the affected upper limb that takes place within 30 min after cessation of the ESS. We expect that brain excitability will be increased during this time interval due to the ESS $[18,23]$. To our knowledge, there is no commonly acknowledged protocol for motor training of upper limb paresis following stroke and, therefore, we decided that the motor training would be provided in accordance to the existing clinical practice at the department which is currently not standardized. Thus, the upper limb training consists of active, repetitive, taskoriented practice. If the trial participant presents a severe upper limb paresis with no or highly limited active movements, the therapist in charge for the particular training session decides which other intervention methods will be employed. A task-oriented exercise bank (see Additional file 2) is available for the physical (PTs) and occupational therapists (OTs) delivering the upper limb training. However, it is a requirement that trial participants receive a minimum of 15 min of arm training following each ESS session. Both groups are offered the same upper limb training.

Usual rehabilitation is defined as $\mathrm{PT}$ and OT training received by the stroke patients in the hospital as well as at rehabilitation centers and, if relevant, at nursing homes in the community during their 6-month trial participation.

\section{Outcome measures}

The primary outcome measure is the Box and Block test (BBT) at 6 months post stroke. The BBT assesses the upper limb's capacity to perform reaching, grasping, moving and releasing objects unilaterally [40]. These motor skills are essential components of performing ADLs and, therefore, of relevance when evaluating whether our intervention contributes to achieving the ultimate goal of rehabilitation: independence in daily life. Normative data are available for the healthy adult population [40], and the instrument has been validated for use in stroke patients [41]. 
The secondary outcomes measures are: (1) the upper extremity section of the Fugl-Meyer Assessment (FMAUE) [42-44], (2) hand-grip strength [45], (3) palmar, lateral and thumb-to-index pinch strength [45], (4) perceptual threshold of touch $[46,47]$, (5) pain in the upper limb during performance of the BBT using Numerical Rating Scale-11 [48] and (6) the mRS score $[49,50]$. The FMA-UE, hand-grip strength, pinch strength, perceptual threshold of touch, and degree of pain are ways to quantify the level of motor and sensory impairments in the upper limb. The mRS is a clinician-reported measure of global disability which is widely used as an endpoint in stroke trials.

All outcome measurements are carried out by EG. Baseline assessments are performed at the stroke unit. Post-intervention and 6-month follow-up assessments are performed at the stroke unit, the patient's home, or the inpatient rehabilitation center or nursing home, depending on the residence of the participant at the scheduled time.

\section{Sample size estimation}

Using a pretrial power analysis, we determined that a minimum sample size of 37 patients was required for correctly detecting a within-group improvement of 5.5 on the BBT, if such a difference truly exists, with a twosided significance level of $5 \%$ and a power of $80 \%$ [51]. The number of 5.5 blocks had previously been reported as the smallest real difference between two measurements for the affected upper limb [41]. Since the minimally clinically important difference on BBT is, to our knowledge, not established yet, we made the assumption that the smallest real difference would be perceived by stroke patients as being of clinical relevance for their daily life. After adjusting for: (a) a case-fatality rate of $8 \%$ as reported at 1 year post stroke in a Danish nationwide population-based study [52] and (b) a 20\% loss of participants at follow-up for other reasons, we estimated that 51 participants were needed in each group [53]. Hence, the total sample size is 102 participants.

\section{Randomization}

Participants are allocated sequentially to either the intervention or the control group using a randomization list constructed by block randomization with variable block size. Randomization is furthermore stratified on: (a) sex and (b) the ability to perform active finger extension at baseline; active finger extension has shown to be a simple and reliable early predictor of recovery of upper limb functioning in stroke patients $[9,54]$. The randomization sequence was generated with the random generator in SAS. The randomization list is kept by administrative personnel and concealed from the other project investigators, with the exception of SPM. Allocations are performed after baseline assessments by contacting the administrative personnel who forwards the group allocation to the personnel responsible for ESS/sham ESS treatment. SPM is occasionally involved in performance of group allocation in the absence of the administrative personnel.

\section{Blinding}

Although complete blinding of the participants to the group allocation is impossible because of the nature of the ESS treatment (i.e., participants can feel the stimulation and are aware of whether it is delivered in continuous or intermittent sham mode), participants are kept unaware of how it is supposed to feel. The personnel who apply ESS are not blinded to the group allocation, and they also collect data from medical records that will be used to characterize the sample. The therapists providing usual rehabilitation as well as other personnel involved in usual patient care are blinded to the group allocation. Investigators who perform outcome assessments and data analysis are unaware of the group allocation, with the exception of the principal investigator (SPM) who is involved in group allocation in the absence of the administrative personnel, who are normally responsible for the group allocation and not involved in any other trial procedures. For further details see Table 1.

\section{Statistical analysis}

Background characteristics will be compared between the intervention groups with $t$ tests (continuous variables) or chi-squared tests (categorical variables). The development of both primary and secondary outcome variables will be analyzed in longitudinal models over the stroke recovery trajectory (baseline, post-intervention and 6-month follow-up), and the difference of the outcomes between the two intervention groups at each of the study time points will be analyzed in multivariable linear regression models. Analyses will be adjusted for the stratification variables (sex and the ability to perform finger extension). Possible differential attrition is adjusted for by weighting the outcomes that are available at each of the study time points with the inverse of the probability of being present; these probabilities are estimated in logistic regression models including all background characteristics and outcomes at previous study time points. To account for this weighting and for repeated observations on the same individual generalized estimating equations (GEE) methods are used to adjust the variance of the parameter estimates. Analyses are performed with SAS version 9.4. The statistical significance level is $5 \%$.

Additional file 3 shows the SPIRIT Checklist $[55,56]$ for this study protocol. 


\section{Discussion}

To our knowledge, this is the first trial investigating the effect of multiple sessions of ESS treatment in the acute stroke phase on the recovery of upper limb paresis. Several studies have shown that the process of upper limb recovery, and especially that of hand dexterity, is most pronounced during the first 4 weeks post stroke after which the recovery gradually levels off before reaching a plateau around 6 months post stroke. Therefore, initiation of rehabilitation in the early weeks post stroke may be essential for achieving successful upper limb recovery at the end of the rehabilitation process. Our trial intends to evaluate the effect of ESS - a rehabilitation method with the potential of applicability in clinical practice. ESS is easy to administer, inexpensive, free of patient discomfort and probably highly acceptable to frail patients in the early days post stroke.

Contrary to previous studies that primarily focused on the stimulation of the peripheral hand nerves at wrist level, we use two sets of large electrodes (see "Intervention" section) to stimulate somatosensory receptors in the shoulder, elbow and wrist regions. We believe that the larger area covered may be beneficial, but because we do not have a third trial arm receiving ESS at wrist level only, we will not be able to identify the effect of increasing the stimulation areas per se.

The eligibility criteria for participation in this trial are very broad. Basically, we include all adult stroke patients living in the hospital's catchment area, except those with remaining upper limb paresis from a previous stroke, contraindications to ESS, severe prestroke disability or inability to provide informed consent. Based on this nonrestrictive participant selection, the results can be generalized to the larger population of patients with a first-ever stroke or successful recovery after a previous stroke and who present an upper limb paresis of varying degrees of severity. However, since our trial is not powered to perform subgroup analyses, we may encounter challenges in detecting the effect of ESS in such a heterogeneous sample. The possibility of overlooking a treatment effect on a specific subgroup of stroke patients (type II error) is a limitation of the study.

Designing the control ESS treatment was challenging since the intervention can be perceived by the trial participants who may also interact with each other during hospitalization. Since a completely inactive ESS treatment gave rise to concerns about high dropout rates in the control group, we designed a sham ESS treatment with an extremely low treatment dose. It is unlikely that the total amount of sham ESS treatment $(1.2 \mathrm{~min})$ is sufficient to induce any training effects. However, we are unable to verify this since we do not have a trial arm receiving completely inactive or no ESS. As a consequence, our trial results can be biased towards an underestimation of the effect of ESS treatment. It is noteworthy that both the control and the intervention group receive the same amount of attention from the personnel during the ESS sessions. Although some participants might figure out their group allocation, we hope that they would be motivated to continue in the trial due to fact that they are offered upper limb training in addition to the usual rehabilitation.

\section{Trial status}

The recruitment of participants was initiated on 13 October 2014 and will continue until complete sample size is achieved which is expected in March 2017. At the submission time of this protocol article, patients are still being recruited for the trial.

\section{Additional files}

Additional file 1: ESS treatment protocol version 2016_09_05. (DOCX $26657 \mathrm{~kb}$ )

Additional file 2: Exercise bank for active, repetitive, task-oriented upper limb training following stroke version 2017_01_22. (PDF 20460 kb)

Additional file 3: The SPIRIT Checklist for the study protocol. (DOC $121 \mathrm{~kb}$ )

Abbreviations

ADLs: Activities of daily living; ES: Electrical stimulation; ESS: Electrical somatosensory stimulation; mRS: Modified Rankin Scale; OT: Occupational therapy/occupational therapist; PT: Physiotherapy/physiotherapist

\section{Acknowledgements}

We would like to thank:

- the Department of Physical and Occupational Therapy for supporting this trial financially and nonfinancially

- all PTs and OTs at the rehabilitation stroke unit for providing upper limb training following ESS/sham ESS and recording the training delivered to the trial participants during their hospitalization

- the nursing staff at the rehabilitation stroke unit for supporting the implementation of study procedures (e.g., ESS/sham ESS treatment, outcome assessments) in daily clinical practice

- all the participating rehabilitation centers (Hjerneskade- og

Rehabiliteringscentret, Rehabiliteringscenter Emdrup, Træningscenter

Bispebjerg, Aktivitetscentret Bispebjerg, Træningscenter Indre By/Østerbro, Rehabiliteringscenter Indre By/Østerbro, Sundhedscentret Stockflethsvej, Frederiksberg Rehabilitering Lioba, Frederiksbergs Kommunes

Rehabiliteringsenhed Valby, Center for Neurorehabilitering Kurhus, Center for Sundhed og Omsorg/Sundhed og Træning Korsør, Sundhed og Genoptræning Amager and the Center for Rehabilitation of Brain Injury) and nursing homes (Ingeborggården, Søndervang, Poppelbo) in the community for recording the PT and OT training provided to our participants during the first 6 months post stroke. A special acknowledgement goes to our contact persons at each of the abovementioned institutions

- the administrative personnel at Bispebjerg Hospital responsible for group allocation

- the Department of Physical and Occupational Therapy at Rigshospitalet/ Glostrup Hospital for introducing OTs/PTs to task-oriented upper limb training

\section{Funding}

The trial is mainly funded by grants from the Capital Region of Denmark, Foundation for Health Research (received on 21 May 2014) and internal financial, administrative and personnel support is received throughout the entire trial from the Department of Physical and Occupational Therapy, Bispebjerg Hospital. Other external grants from Bevica Fonden (received on 17 December 2013), Lundbeckfonden (FP 68/2013), the Danish Association of Occupational Therapists (FF 1 14-3) and Direktør Jacob Madsen and his 
wife, Olga Madsen's Fund (5507) also contributed to the funding of this trial. The funding sources have neither influence on the trial design, data collection, management, analysis, interpretation, nor on reporting of the results.

\section{Availability of data and materials}

After the trial is completed and the results are disseminated in peerreviewed journals, the datasets generated and/or analyzed during the present trial will be deposited and publicly available at the Danish National Archives. While the trial is still ongoing, the datasets are available from the corresponding author on reasonable request.

\section{Authors' contributions}

EG initiated and designed the trial, applied for funding, established collaborative relationships with participating centers in the community, is responsible for trial logistics, recruitment of participants, data collection and entry, outcome assessments, and has prepared the first version of this manuscript. CC developed the concept, designed the trial, assisted with establishing relationships with participating centers in the community, developed the ESS treatment protocol, and has critically revised this manuscript. CH described the ESS treatment protocol and test procedures relating to the treatment, was responsible for the collaboration with the clinical hospital staff, for coordinating and executing the ESS treatment during the first year, and for the training and supervision of the additional ESS personnel. VS contributed to trial design and statistical assistance, and has critically revised this manuscript. MS contributed to trial conduct within the Department of Neurology and to the collection of medical data. HC contributed to trial design and conduct within the Department of Neurology and has critically revised this manuscript. SPM developed the concept, designed the trial and edited the manuscript. All authors have read and approved the final version of the manuscript.

\section{Competing interests}

The authors declare that they have no competing interests.

\section{Consent for publication}

Written informed consent was obtained for publication of the accompanying images in this manuscript. The Consent Form is held by the corresponding author and is available for review by the Editor-in-Chief.

\section{Ethics approval and consent to participate}

The trial was approved by the Capital Region of Denmark's Committee on Health Research Ethics (H-4-2014-012) on 2 October 2014 and by the Danish Data Protection Agency (2012-58-0004) on 21 May 2014. The trial protocol was notified at ClinicalTrials.gov (NCT02250365) on 18 September 2014. Informed consent was collected from each participant before the initiation of any trial procedures.

\section{Author details}

'Department of Physical and Occupational Therapy, Bispebjerg Hospital, Bispebjerg Bakke 23, bldg. 10, 2400, Copenhagen, Denmark. 'Faculty of Health and Medical Sciences, University of Copenhagen, Copenhagen, Denmark. ${ }^{3}$ Institute of Sports Medicine, Department of Orthopaedic Surgery M, Bispebjerg Hospital, Copenhagen, Denmark. ${ }^{4}$ Center for Healthy Aging, Faculty of Health and Medical Sciences, University of Copenhagen, Copenhagen, Denmark. ${ }^{5}$ The Research Unit for General Practice and Section of General Practice, Department of Public Health, University of Copenhagen, Copenhagen, Denmark. ${ }^{6}$ Department of Neurology, Bispebjerg Hospital, Copenhagen, Denmark.

Received: 4 November 2016 Accepted: 24 January 2017 Published online: 23 February 2017

\section{References}

1. Murray CJ, Barber RM, Foreman KJ, Abbasoglu Ozgoren A, Abd-Allah F, Abera SF, et al. Global, regional, and national disability-adjusted life years (DALYS) for 306 diseases and injuries and healthy life expectancy (HALE) for 188 countries, 1990-2013: quantifying the epidemiological transition. Lancet. 2015;386(10009):2145-91.

2. Camak DJ. Addressing the burden of stroke caregivers: a literature review. J Clin Nurs. 2015;24(17-18):2376-82.
3. Jennum P, Iversen HK, Ibsen R, Kjellberg J. Cost of stroke: a controlled national study evaluating societal effects on patients and their partners. BMC Health Serv Res. 2015;15:466.

4. Feigin VL, Barker-Collo S, McNaughton H, Brown P, Kerse N. Long-term neuropsychological and functional outcomes in stroke survivors: current evidence and perspectives for new research. Int J Stroke. 2008;3(1):33-40.

5. Nakayama H, Jorgensen HS, Raaschou HO, Olsen TS. Recovery of upper extremity function in stroke patients: the Copenhagen Stroke Study. Arch Phys Med Rehabil. 1994;75(4):394-8.

6. Lawrence ES, Coshall C, Dundas R, Stewart J, Rudd AG, Howard R, et al. Estimates of the prevalence of acute stroke impairments and disability in a multiethnic population. Stroke. 2001;32(6):1279-84.

7. Persson HC, Parziali M, Danielsson A, Sunnerhagen KS. Outcome and upper extremity function within 72 hours after first occasion of stroke in an unselected population at a stroke unit. A part of the SALGOT study. BMC Neurol. 2012;12:162.

8. Sveen U, Bautz-Holter E, Sodring KM, Wyller TB, Laake K. Association between impairments, self-care ability and social activities 1 year after stroke. Disabil Rehabil. 1999;21(8):372-7.

9. Nijland RH, van Wegen EE. Harmeling-van der Wel BC, Kwakkel G. Presence of finger extension and shoulder abduction within 72 hours after stroke predicts functional recovery: early prediction of functional outcome after stroke: the EPOS cohort study. Stroke. 2010;41(4):745-50.

10. Kwakkel G, Kollen BJ, van der Grond J, Prevo AJ. Probability of regaining dexterity in the flaccid upper limb: impact of severity of paresis and time since onset in acute stroke. Stroke. 2003;34(9):2181-6.

11. Fleming MK, Newham DJ, Roberts-Lewis SF, Sorinola IO. Self-perceived utilization of the paretic arm in chronic stroke requires high upper limb functional ability. Arch Phys Med Rehabil. 2014;95(5):918-24.

12. Wade DT, Langton-Hewer R, Wood VA, Skilbeck CE, Ismail HM. The hemiplegic arm after stroke: measurement and recovery. J Neurol Neurosurg Psychiatry. 1983;46(6):521-4

13. Verheyden G, Nieuwboer A, De Wit L, Thijs V, Dobbelaere J, Devos H, et al. Time course of trunk, arm, leg, and functional recovery after ischemic stroke. Neurorehabil Neural Repair. 2008;22(2):173-9.

14. Au-Yeung SS, Hui-Chan CW. Predicting recovery of dextrous hand function in acute stroke. Disabil Rehabil. 2009;31(5):394-401.

15. Popovic DB, Sinkaer T, Popovic MB. Electrical stimulation as a means for achieving recovery of function in stroke patients. NeuroRehabilitation. 2009; 25(1):45-58

16. Ridding MC, Brouwer B, Miles TS, Pitcher JB, Thompson PD. Changes in muscle responses to stimulation of the motor cortex induced by peripheral nerve stimulation in human subjects. Exp Brain Res. 2000;131(1):135-43.

17. Ridding MC, McKay DR, Thompson PD, Miles TS. Changes in corticomotor representations induced by prolonged peripheral nerve stimulation in humans. Clin Neurophysiol. 2001;112(8):1461-9.

18. Kaelin-Lang A, Luft AR, Sawaki L, Burstein AH, Sohn YH, Cohen LG. Modulation of human corticomotor excitability by somatosensory input. J Physiol. 2002;540(Pt 2):623-33.

19. McKay D, Brooker R, Giacomin P, Ridding M, Miles T. Time course of induction of increased human motor cortex excitability by nerve stimulation. Neuroreport. 2002;13(10):1271-3.

20. Wu CW, van Gelderen P, Hanakawa T, Yaseen Z, Cohen LG. Enduring representational plasticity after somatosensory stimulation. Neuroimage. 2005;27(4):872-84

21. Tinazzi M, Zarattini S, Valeriani M, Romito S, Farina S, Moretto G, et al. Longlasting modulation of human motor cortex following prolonged transcutaneous electrical nerve stimulation (TENS) of forearm muscles: evidence of reciprocal inhibition and facilitation. Exp Brain Res. 2005;161(4):457-64.

22. Golaszewski SM, Siedentopf CM, Koppelstaetter F, Rhomberg P, Guendisch GM Schlager A, et al. Modulatory effects on human sensorimotor cortex by wholehand afferent electrical stimulation. Neurology. 2004;62(12):2262-9.

23. Golaszewski SM, Bergmann J, Christova M, Nardone R, Kronbichler M, Rafolt $\mathrm{D}$, et al. Increased motor cortical excitability after whole-hand electrical stimulation: a TMS study. Clin Neurophysiol. 2010;121(2):248-54.

24. Dobkin BH. Do electrically stimulated sensory inputs and movements lead to long-term plasticity and rehabilitation gains? Curr Opin Neurol. 2003;16(6):685-91.

25. Koesler IB, Dafotakis M, Ameli M, Fink GR, Nowak DA. Electrical somatosensory stimulation improves movement kinematics of the affected hand following stroke. J Neurol Neurosurg Psychiatry. 2009;80(6):614-9. 
26. Conforto AB, Kaelin-Lang A, Cohen LG. Increase in hand muscle strength of stroke patients after somatosensory stimulation. Ann Neurol. 2002;51(1):122-5.

27. Sawaki L, Wu CW, Kaelin-Lang A, Cohen LG. Effects of somatosensory stimulation on use-dependent plasticity in chronic stroke. Stroke. 2006;37(1):246-7.

28. Klaiput A, Kitisomprayoonkul W. Increased pinch strength in acute and subacute stroke patients after simultaneous median and ulnar sensory stimulation. Neurorehabil Neural Repair. 2009;23(4):351-6.

29. Celnik P, Hummel F, Harris-Love M, Wolk R, Cohen LG. Somatosensory stimulation enhances the effects of training functional hand tasks in patients with chronic stroke. Arch Phys Med Rehabil. 2007;88(11):1369-76.

30. Wu CW, Seo HJ, Cohen LG. Influence of electric somatosensory stimulation on paretic-hand function in chronic stroke. Arch Phys Med Rehabil. 2006;87(3):351-7.

31. Conforto AB, Cohen LG, dos Santos RL, Scaff M, Marie SK. Effects of somatosensory stimulation on motor function in chronic cortico-subcortical strokes. J Neurol. 2007;254(3):333-9.

32. Conforto AB, Ferreiro KN, Tomasi C, dos Santos RL, Moreira VL, Marie SK, et al. Effects of somatosensory stimulation on motor function after subacute stroke. Neurorehabil Neural Repair. 2010;24(3):263-72.

33. Ikuno K, Kawaguchi S, Kitabeppu S, Kitaura M, Tokuhisa K, Morimoto S, et al. Effects of peripheral sensory nerve stimulation plus task-oriented training on upper extremity function in patients with subacute stroke: a pilot randomized crossover trial. Clin Rehabil. 2012;26(11):999-1009.

34. Dos Santos-Fontes RL, de Ferreiro Andrade KN, Sterr A, Conforto AB. Homebased nerve stimulation to enhance effects of motor training in patients in the chronic phase after stroke: a proof-of-principle study. Neurorehabil Neural Repair. 2013;27(6):483-90.

35. Laufer $Y$, Elboim-Gabyzon M. Does sensory transcutaneous electrical stimulation enhance motor recovery following a stroke? A systematic review. Neurorehabil Neural Repair. 2011;25(9):799-809.

36. Bispebjerg Hospital. About Bispebjerg Hospital. [cited 29 August 2016] Available from: https://www.bispebjerghospital.dk/english/Sider/ default.aspx. Accessed 4 Nov 2016.

37. Watson Te. Electrotherapy. Evidence-based practice. 12th ed.: Elsevier; 2008.

38. Adams Jr HP, Bendixen BH, Kappelle LJ, Biller J, Love BB, Gordon DL, et al. Classification of subtype of acute ischemic stroke. Definitions for use in a multicenter clinical trial. TOAST. Trial of Org 10172 in Acute Stroke Treatment. Stroke. 1993;24(1):35-41

39. Multicenter trial of hemodilution in ischemic stroke-background and study protocol. Scandinavian Stroke Study Group. Stroke. 1985;16(5):885-90

40. Mathiowetz V, Volland G, Kashman N, Weber K. Adult norms for the Box and Block Test of manual dexterity. Am J Occup Ther. 1985;39(6):386-91.

41. Chen HM, Chen CC, Hsueh IP, Huang SL, Hsieh CL. Test-retest reproducibility and smallest real difference of 5 hand function tests in patients with stroke. Neurorehabil Neural Repair. 2009;23(5):435-40.

42. Fugl-Meyer AR. Post-stroke hemiplegia assessment of physical properties. Scand J Rehabil Med Suppl. 1980;7:85-93.

43. Gladstone DJ, Danells CJ, Black SE. The Fugl-Meyer Assessment of motor recovery after stroke: a critical review of its measurement properties. Neurorehabil Neural Repair. 2002;16(3):232-40.

44. Arya KN, Verma R, Garg RK. Estimating the minimal clinically important difference of an upper extremity recovery measure in subacute stroke patients. Top Stroke Rehabil. 2011;18 Suppl 1:599-610.

45. Mathiowetz V, Weber K, Volland G, Kashman N. Reliability and validity of grip and pinch strength evaluations. J Hand Surg [Am]. 1984;9(2):222-6.


(PTT) with high-frequency transcutaneous electric nerve stimulation (Hf/TENS) in elderly patients with stroke: a reliability study. Clin Rehabil. 2003;17(8):825-34

47. Eek E, Holmqvist LW, Sommerfeld DK. Adult norms of the perceptual threshold of touch (PTT) in the hands and feet in relation to age, gender, and right and left side using transcutaneous electrical nerve stimulation. Physiother Theory Pract. 2012;28(5):373-83.

48. Hjermstad MJ, Fayers PM, Haugen DF, Caraceni A, Hanks GW, Loge JH, et al. Studies comparing Numerical Rating Scales, Verbal Rating Scales, and Visual Analogue Scales for assessment of pain intensity in adults: a systematic literature review. J Pain Symptom Manage. 2011;41(6):1073-93.

49. Banks $J$, Marotta CA. Outcomes validity and reliability of the modified Rankin scale: implications for stroke clinical trials: a literature review and synthesis. Stroke. 2007;38(3):1091-6.
50. Bruno A, Shah N, Lin C, Close B, Hess DC, Davis K, et al. Improving modified Rankin Scale assessment with a simplified questionnaire. Stroke. 2010;41(5):1048-50.

51. University of California, San Francisco. Power and sample size programmes. [cited 28 September 2016]. Available from: https://www.stat.ubc.ca/ rollin/ stats/ssize/. Accessed 4 Nov 2016.

52. Langagergaard $V$, Palnum KH, Mehnert F, Ingeman A, Krogh BR, Bartels P, et al. Socioeconomic differences in quality of care and clinical outcome after stroke: a nationwide population-based study. Stroke. 2011;42(10):2896-902.

53. Kirkwood BR SJ. Essential medical statistics. 2nd ed.: Blackwell Science; 2003.

54. Smania N, Paolucci S, Tinazzi M, Borghero A, Manganotti P, Fiaschi A, et al. Active finger extension: a simple movement predicting recovery of arm function in patients with acute stroke. Stroke. 2007;38(3):1088-90.

55. Chan AW, Tetzlaff JM, Altman DG, Laupacis A, Gotzsche PC, Krleza-Jeric K, et al. SPIRIT 2013 statement: defining standard protocol items for clinical trials. Ann Intern Med. 2013;158(3):200-7.

56. Chan AW, Tetzlaff JM, Gotzsche PC, Altman DG, Mann H, Berlin JA, et al. SPIRIT 2013 explanation and elaboration: guidance for protocols of clinical trials. BMJ. 2013;346:e7586

\section{Submit your next manuscript to BioMed Central and we will help you at every step:}

- We accept pre-submission inquiries

- Our selector tool helps you to find the most relevant journal

- We provide round the clock customer support

- Convenient online submission

- Thorough peer review

- Inclusion in PubMed and all major indexing services

- Maximum visibility for your research

Submit your manuscript at www.biomedcentral.com/submit

) Biomed Central 